\title{
Radioguided occult lesion localisation (ROLL) in surgery of impalpable breast tumours
}

\author{
M. H. K. Leidenius \\ Breast Surgery Unit, Helsinki University Central Hospital, Helsinki, Finland.
}

\begin{abstract}
With advanced detection methods now allowing cancers to be found at earlier and earlier stages, new treatment methods to deal with these early stage cancers are also emerging. Radioguided occult lesion localisation (ROLL), a technique pioneered in 1996, is a useful method for localisation of impalpable breast tumours with results superior to those obtained by wire localisation and comparable to those achieved by surgery of palpable lesions. ROLL allows for the precise localisation necessary to optimise the cosmetic outcome in breast conserving surgery without jeopardising sufficient free tissue margins. ROLL can also be used in conjunction with sentinel node biopsy (SNB) using either the same or different radioactive tracer. For all these reasons, ROLL is an important development in breast cancer treatment.
\end{abstract}

Keywords: Nonpalpable lesions; Preinvasive lesions; Radioguided occult lesion localisation; ROLL; Tumour localisation

\section{Introduction}

The widespread use of breast imaging techniques as well as mammographic screening has remarkably increased the proportion of impalpable invasive and preinvasive lesions detected during the last 10 years. Precise localisation of the clinically occult lesion is critical in order to optimise the cosmetic outcome in breast conserving surgery without jeopardising sufficient free tissue margins. Detecting, diagnosing and treating these tumours is a surgical challenge.

Traditionally, the localisation of the tumour is done by guidance of a wire inserted under radiographic control [1]. The insertion of a wire may be technically difficult leading to a imprecise wire placement. Migration, displacement or transection of the wire may occur. Furthermore, the procedure is uncomfortable for the patient, and the wire may also harm a

Correspondence to: Marjut H. K. Leidenius, Breast Surgery Unit, Helsinki University Central Hospital, P.O. Box 140, 00029 HUS, Helsinki, Finland. E-mail: marjut.leidenius@hus.fi; Tel: +358 50 4271005; Fax: +358947176301

Publication date 30/06/05 $\mathrm{BCO} / 348 / 2005 / \mathrm{FO}$ member of the surgical team or the pathologist examining the resection specimen.

Radioguided occult lesion localisation (ROLL) has emerged as a novel technique in surgery of impalpable breast lesions inspired by sentinel node biopsy (SNB) [2]. In ROLL, radioisotope injection adjacent to the lesion is performed under ultrasonographic or stereotactic guidance preoperatively. Intraoperative tumour localisation is carried out searching for the maximal radioactivity by gamma-probe designed for sentinel lymph node localisation.

\section{The technique}

The technique was pioneered in 1996 at the European Institute of Oncology, Milan, applying an intratumoural injection of Tc99m-labelled human albumin colloid with particle size large enough to retain at the injection site $\left(\right.$ Macrotec $^{\circledR}$ ) [2]. In order to ensure the exact placement of the injection and to assist the surgeon in locating the lesion, breast scintigraphy superimposing the scintigram on the mammogram was to be performed [2]. Alternatively, an additional injection or contrast medium subsequent to the radioisotope 
has been injected to confirm the position by preoperative mammography [3]. Recently, the original technique has been simplified performing the localisation without breast scintigraphy or contrast medium injection and mammography $[4,5]$. However, it is critical that the lesion is undisputedly recognisable in breast ultrasonography, when using an ultrasonographically guided radioisotope injection without breast scintigram or mammogram. ROLL has also been performed in combination with wire guidance [6] or placing a titanium seed containing iodine-125, commonly used for the brachytherapy of prostate cancer, adjacent to the tumour $[7,8]$.

The centring of the tumour with a hand-held gamma-probe under radioactive guidance helps the removal of the lesion with adequate uniform margin of healthy tissue. The tumour edges are easily controlled using a gamma-probe while the radioactivity decreases clearly outside the tumour. Furthermore it is easy to check residual radioactivity from the wound to ensure that the tumour is excised radically. The control of margins using wire localisation is more difficult because of wire displacement or migration or when the tip of the guide wire is distant from the lesion after an unsuccessful wire placement.

\section{The outcome}

The localisation time either using ultrasonography or stereotactic guidance has been found significantly reduced compared with guide wire placement [9]. However, the length of the surgical procedure may not to reduced with ROLL $[8,9]$. As regards to the radiation exposure, ROLL seems safe to the patient as well as to the medical staff due to the very short half-life of Tc99m of only $6 \mathrm{~h}$, and the low dose gamma radiation used [10].

All studies evaluating ROLL has concluded it as easy and accurate method for a surgeon to find an occult breast lesion and excise it adequate free tissue margins [2-9,11,12]. According to our experience, tumour localisation has been successful in all cases and at least $4 \mathrm{~mm}$ free margins have been achieved in over $90 \%$ of cases after the completion of the primary operation [4]. ROLL has been found as [8,9] superior to the wire guided resection as regards to achieved free tissue margins. ROLL also enables reduced excision volume [9] and better lesion centring [11] within the specimen compared to wire localisation. Consequently, even the cosmetic results seem better when using ROLL [9]. Furthermore, the results achieved by ROLL have been fully comparable to those obtained in breast conserving for palpable cancer as regards to sufficient free tissue margins as well as the size of the excised specimen related to the tumour size [4].

\section{ROLL and simultaneous SNB}

A few studies have addressed the feasibility of ROLL in connection with lymphoscintigraphy and SNB $[3,6,7,12]$. ROLL has been performed using radioactive seed localisation and the simultaneous SNB applying peritumoral injection of Tc99m labelled sulphur colloid [7]. Alternatively, a subdermal injection of Tc99m labelled small particle human albumin colloid $\left(\right.$ Nanocoll $\left.{ }^{\circledR}\right)$ may be used for SNB and an intratumoural injection of Tc99m colloid with the larger particle size (Maasol ${ }^{\circledR}$ ) for ROLL [12]. Furthermore, ROLL and SNB have been performed also using the same radioisotope (Nanocoll ${ }^{\circledR}$ ) injecting half of the dose of intratumourally for ROLL and the other half more superficially to facilitate the transportation to the lymph nodes [3] or even applying a single intratumoral tracer injection for both ROLL and SNB $[4,6]$. Simultaneous performance of ROLL and SNB seems very feasible in the management of early breast cancer.

\section{Concluding remarks}

ROLL is a useful method for localisation of impalpable breast tumours with results superior to those obtained by wire localisation and comparable to those achieved by surgery of palpable lesions. Furthermore, ROLL is feasible in connection with SNB using either the same or different radioactive tracer.

\section{References}

1. Perry NM. Quality assurance in the diagnosis of breast disease. Eur J Cancer 2001; 37: 159-172.

2. Luini A, Zurrida S, Galimberti V, Paganelli G. Radioguided surgery of occult breast lesions. Eur J Cancer 1998; 34: 204-205.

3. Feggi L, Basaglia E, Corcione S, et al. An original approach in the diagnosis of early breast cancer: use of the same radiopharmaceutical for both non-palpable lesions and sentinel node localisation. Eur J Nucl Med 2001; 28: 1589-1596.

4. Ronka R, Krogerus L, Leppanen E, von Smitten K, Leidenius M. Radio-guided occult lesion localization in patients undergoing breast-conserving surgery and sentinel node biopsy. Am J Surg 2004; 187: 491-496.

5. Audisio RA, Nadeem R, Harris O, Desmond S, Thind R, Chagla LS. Radioguided occult lesion localisation (ROLL) is available in the UK for impalpable breast lesions. Ann R Coll Surg Engl 2005; 87: 92-95.

6. Tanis PJ, Deurloo EE, Valdes Olmos RA, et al. Single intralesional tracer dose for radio-guided excision of clinically occult breast cancer and sentinel node. Ann Surg Oncol 2001; 8: 850-855.

7. Gray RJ, Giuliano R, Dauway EL, Cox CE, Reintgen DS. Radioguidance for nonpalpable primary lesions and sentinel lymph node(s). Am J Surg 2001; 182: 404-406.

8. Gray RJ, Salud C, Nguyen K, et al. Randomized prospective evaluation of a novel technique for biopsy or 
lumpectomy of nonpalpable breast lesions: radioactive seed versus wire localization. Ann Surg Oncol 2001; 8: 711-715.

9. Nadeem R, Chagla L, Harris O, et al. Occult breast lesions: a comparison between radioguided occult lesion localisation (ROLL) vs wire guided lumpectomy (WGL). Breast, in press.

10. Cremonesi M, Ferrari M, Sacco E, et al. Radiation protection in radioguided surgery of breast cancer. Nucl Med Commun 1999; 20: 919-924.
11. Luini A, Zurrida S, Paganelli G, et al. Comparison of radioguided excision with wire localization of occult breast lesions. Br J Surg 1999; 86: 522-555.

12. De Cicco C, Trifiro G, Intra M, et al. Optimised nuclear medicine method for tumour marking and sentinel node detection in occult primary breast lesions. Eur $\mathrm{J} \mathrm{Nucl}$ Med Mol Imaging 2004; 31: 349-354. 